\title{
The limits of inelastic tunneling spectroscopy for identifying transport pathways
}

\author{
Michael Deffner and Carmen Herrmann* \\ Department of Chemistry, University of Hamburg, Germany, Martin-Luther-King-Platz 6, \\ 20146 Hamburg \\ E-mail: carmen.herrmann@chemie.uni-hamburg.de
}

\begin{abstract}
Inelastic Electron Tunneling Spectroscopy (IETS) is a powerful tool to study the properties of molecular junctions. In particular, it is considered useful for extracting information on electron transport pathways. We explore the limits of this approach by comparing computed interatomic transmission pathways with IETS intensities for different molecular junctions, employing a new efficient implementation for evaluating IETS intensities via the mode-tracking algorithm. We find that while a correlation between pathways and IETS intensities indeed holds when vibrations are clearly localized on atoms off the transport pathway, there is no such correlation for molecules with less localized vibrations, even if transport pathways only sample part of the molecule, and even if a statistical analysis over the vibrational modes is made. This could indicate that the significance of IETS signals for transport pathways is limited to molecules with localized vibrational modes.
\end{abstract}


Electron transport through individual molecules is important for many chemical and biological processes, as well as for potential technological applications. Experimental and theoretical progress in this field in recent years has also provided fundamental insight into molecules under unusual (nonequilibrium) conditions. ${ }^{1-122}$ For understanding and controlling electron transport through molecules, it is important to know which path an electron follows through a molecule. This has been studied extensively in proteins,, $13-17$ and pathway control has been achieved via infrared excitations in donor-bridge-acceptor systems. ${ }^{18}$ Pathway analyses are also essential for identifying whether electrons follow helical paths in helixshaped molecules ${ }^{\sqrt{19}}$ or in electronically helical structures, $\stackrel{20]}{{ }^{20}}$ wich may be important for

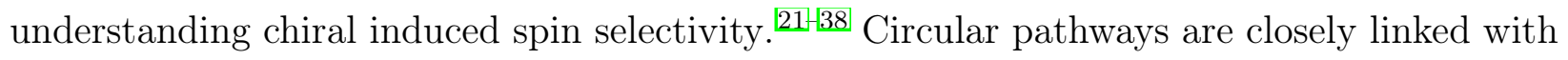
destructive quantum interference in molecular junctions,, 399 and engineering pathways can be employed as a means of manipulating the transport properties of molecular systems. ${ }^{42}$

While a number of theoretical approaches exist for extracting pathway information, 911411512013943151 access by experimental methods is limited. An experimental technique which is able to provide pathway information is inelastic tunneling spectroscopy (IETS), tures of tunneling electrons exciting molecular vibrations are recorded. In addition to a set

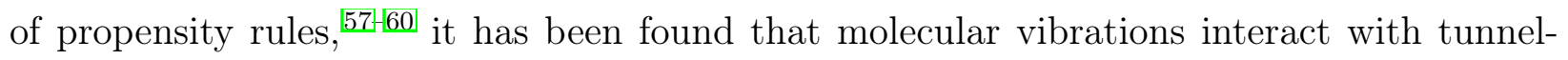
ing electrons much more strongly when they are located within their path than when they are outside of it. ${ }^{[5253]}$ We explore the limits of this relation by comparing electron transport pathways and IETS intensities from first-principles simulations not only for systems with well-localized vibrations, but also with delocalized ones. We find that for the latter case (which is frequent in typical molecular junctions), IETS cannot give information on transport pathways, even if a statistical analysis over vibrational modes is made.

For short molecules off resonance and at low temperatures, the dominant transport mechanism usually is elastic coherent tunneling. ${ }^{115461}$ Aside from elastic processes, inelastic ones such as excitations into different spin or vibrational states can be measured in a scanningtunneling-microscopy (STM) or break-junction experiment at low temperatures, $\frac{16263}{16 p-}$ 
pearing as a peak or dip in the second derivative of the current $I$ with respect to the voltage $V$. When the applied voltage provides enough energy to excite a vibration, an additional transport channel is opened as shown in Figure 1. Thus, a complete vibrational spectrum of the junction can be obtained by varying the applied voltage.

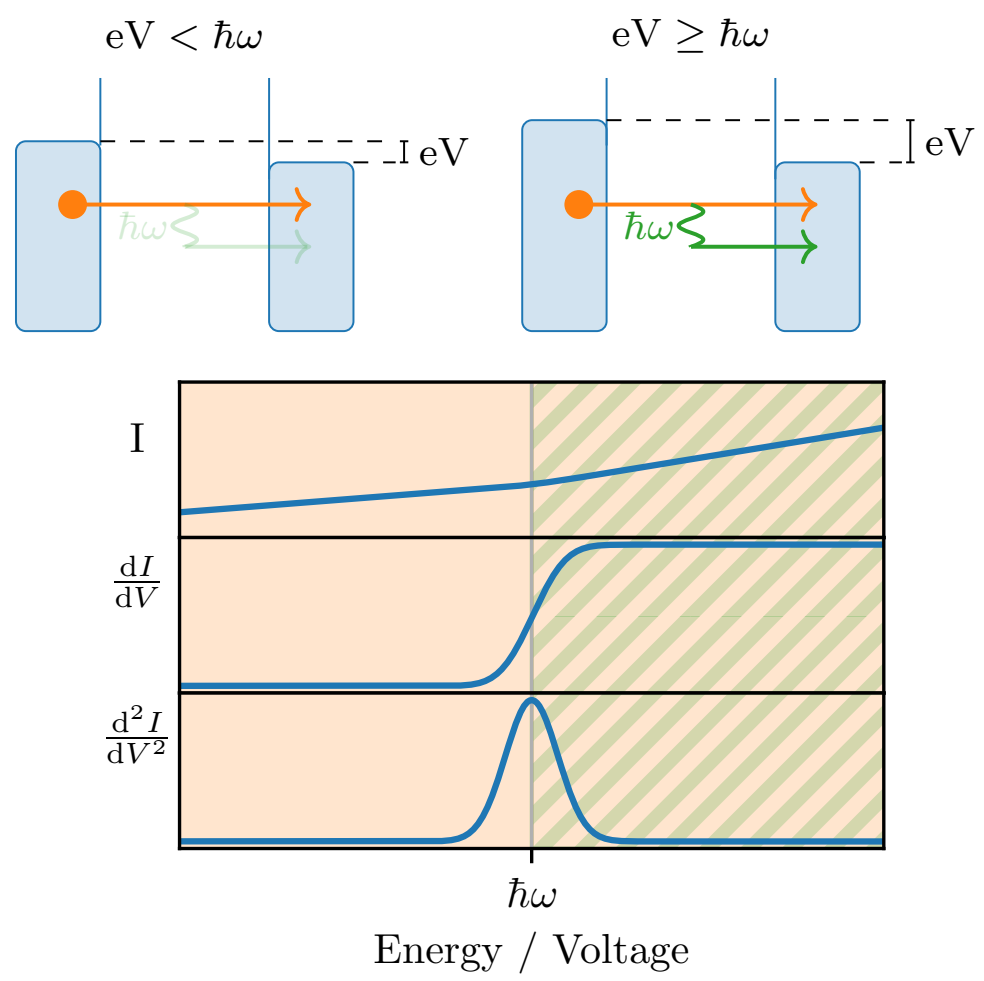

Figure 1: Elastic and inelastic tunneling through a tunneling barrier representing a molecule between two electrodes. $\hbar \omega$ corresponds to the energy of a vibrational quantum. For energies below $\hbar \omega$, just the elastic channel is available. At energies higher or equal to $\hbar \omega$, the inelastic channel is opened, which leads to a slight increase of the tunneling current. By calculating the second derivative of the current with respect to the voltage, the IET spectrum is obtained.

For the calculation of IET spectra, several approaches have been suggested. ${ }^{64}[69$ We follow the approach derived by Troisi et. al., ${ }^{69}$ who generalized the theoretical description of the elastic tunneling current through a molecular junction within the coherent tunneling regime. $.70[72$

Using a Green's function approach, $\frac{\sqrt{73}}{\sqrt{3}}$ the transmission function $T(E)$ is obtained as

$$
T(E)=\operatorname{tr}\left(\boldsymbol{\Gamma}_{\mathrm{L}}(E) \boldsymbol{G}_{\mathrm{C}}(E) \boldsymbol{\Gamma}_{\mathrm{R}}(E) \boldsymbol{G}_{\mathrm{C}}(E)^{\dagger}\right) .
$$


This roughly describes the probability of an electron to be transmitted from one electron to the other at a certain energy. The elastic zero-bias conductance $g$ is given by

$$
g=g_{0} T\left(E_{\mathrm{F}}\right)
$$

$\boldsymbol{\Gamma}_{\mathrm{L}}, \boldsymbol{\Gamma}_{\mathrm{R}}$ describe the electronic coupling between the left or right electrode and the molecular subsystem, while $\boldsymbol{G}_{\mathrm{C}}$ is the Green's matrix describing the molecule (or more generally the central region) in the presence of the electrodes. $g_{0}=\frac{2 e^{2}}{h}$ is the quantum of conductance (with $e$ the unit charge and $h$ Planck's constant).

To introduce electron-phonon coupling, the dependence of the central-region Green's function on the nuclear coordinates is taken into account. Performing a Taylor expansion of $\boldsymbol{G}_{\mathrm{C}}$ for a set of vibrational modes $\left\{Q_{\alpha}\right\}$ in the harmonic approximation (using mass-weighted coordinates) around the equilibrium structure, the first-order correction is nonzero only for an initial and final state, which differ by one vibrational quantum. ${ }^{69}$ This part $\left(\boldsymbol{G}_{C}^{\alpha}\right)$ describes the correction to the elastic current due to the emission (or adsorption) of one vibrational quantum and is given by

$$
G_{i j}^{\alpha}=\frac{\sqrt{2}}{2}\left(\frac{\partial G_{i j}}{\partial Q_{\alpha}}\right)_{0}
$$

for each matrix element. From this matrix, the peak area for each mode $\alpha$ can be calculated by $\frac{75}{75}$

$$
W_{\alpha}=g_{0} \operatorname{tr}\left(\boldsymbol{\Gamma}_{\mathrm{L}}\left(E_{\mathrm{F}}\right) \boldsymbol{G}_{\mathrm{C}}^{\alpha}\left(E_{\mathrm{F}}\right) \boldsymbol{\Gamma}_{\mathrm{R}}\left(E_{\mathrm{F}}\right) \boldsymbol{G}_{\mathrm{C}}^{\alpha}\left(E_{\mathrm{F}}\right)^{\dagger}\right)
$$

where each matrix is evaluated at the Fermi energy.

Intuitively, one would expect this IETS intensity to be larger for vibrations involving atoms in the path of the tunneling electrons. Therefore, if, for example, the intensity of a vibration localized on a side group is low, this suggests that the current bypasses that 
sidegroup. If vibrational modes are delocalized over substantial parts of the molecule, as is often the case for typical molecular wires studied in conductance experiments, it is less clear how to extract information on current pathways from IET spectra. Inspired by Ref., ${ }^{[52}$ we employ a statistical average in such cases.

Electron tunneling paths are not directly accessible in experiments on molecular junctions, but can be evaluated from theoretical analysis. For many different types of molecules, a decomposition of the overall transmission into atom-to-atom contributions $T_{A B}(E)$ has proven valuable to understand properties like switching or quantum interference $\mathrm{e}^{3946 / 48 / 76}$ (see Supporting Information for more details).

In the following, we compare this information on transmission pathways with IETS intensities of vibrations located on and off the transmission path for three molecules with increasing delocalization of vibrational modes. We treat the Fermi energy as an adjustable parameter for two reasons: (1) It is not clear how to properly choose this energy in a simulation, given that the electrode surfaces in a molecular junction may be irregular and strongly modified by adsorbed molecules, making it difficult to find a good atomistic model, and that standard DFT is not very good at describing molecule-metal surfaces, $\frac{8283}{83}$ and (2) we are interested in general relationships between IETS intensities and electron pathways, so by varying $E_{\mathrm{F}}$, we get more robust information on this relationships from each single molecule than from a single energy value. Such variations of $E_{F}$ can also be thought of as mimicking how chemical substituents can shift transmission curves w.r.t. the Fermi energy of the electrodes. $\frac{8485}{6 h e n}$ varying the Fermi energy, care must be taken when approaching resonances, as the theoretical approach employed for evaluating IETS intensities is based on perturbation theory, and since the Landauer approach may fail in resonance. ${ }^{61}$ However, changes observed when going towards the resonances from a clearly off-resonant situation may be considered reasonably safe to be interpreted in the discussion below.

First, we compare IET spectra and transmission pathways for a system with a welllocalized vibration: an iron(II) porphyrin molecular junction with a $\mathrm{CO}$ and an imidazole 
ligand in is low-spin ground state, inspired by myoglobin (see Figure 2). Porphyrin complexes are of high interest for molecular electronics, $\frac{86}{26}$ for example due to their switching behavior. $\stackrel{8788}{ }$ For the complex chosen here, transmission was previously shown based on theoretical analysis to bypass the central iron atom (and hence the CO ligand) for all energies except close to (sharp) resonances around the iron $d$-orbital energies. $\stackrel{43}{~ T h i s ~ w o u l d ~ s u g g e s t ~}$ that IETS intensities of the CO vibration should be low.

Figure 2 shows the system together with the total transmission, the local transmissions at -5.0 and $-6.0 \mathrm{eV}$ and the summed-up local transmissions involving the central iron atom, the carbon and oxygen atoms of the adsorbed $\mathrm{CO}$, and an anchoring sulfur atom, respectively, normalized by the total transmission,

$$
T_{A}(E)=\frac{\sum_{B}\left|T_{A B}(E)\right|}{T(E)}
$$

where the sum runs over all other atoms $B$. The contribution of the sulfur atom is flat and close to one for all energies, since current will mostly enter or leave the system through the anchoring groups. For the Fe atom and for the CO ligand, the contribution is very low except for some of the energies at which sharp dips or peaks occur, related to destructive quantum

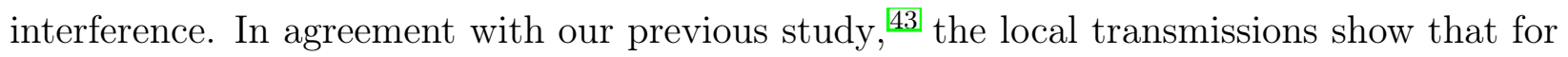
almost all energies, the electron tunnels via the organic backbone around the metal center, while for, e.g., $-6 \mathrm{eV}$ ring currents occur heavily involving the Fe atom. In these regions, the sum over the atomic contributions is occasionally higher than the total transmission, since ring currents can give rise to large contributions for the atom-to-atom transmissions, while the overall through-molecule transmission is low. The calculated IETS intensity for the CO stretching mode $\left(\nu_{C O}=1980.37 \mathrm{~cm}^{-1}\right)$ as a function of energy correlates well qualitatively with the normalized local transmissions through the CO ligand (Figure 2 e)). 


\section{a)}

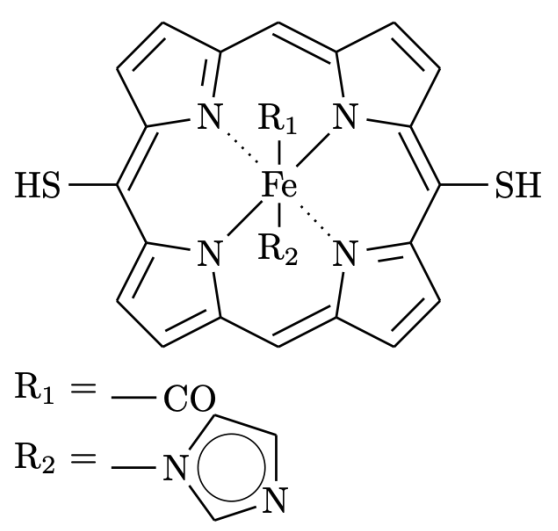
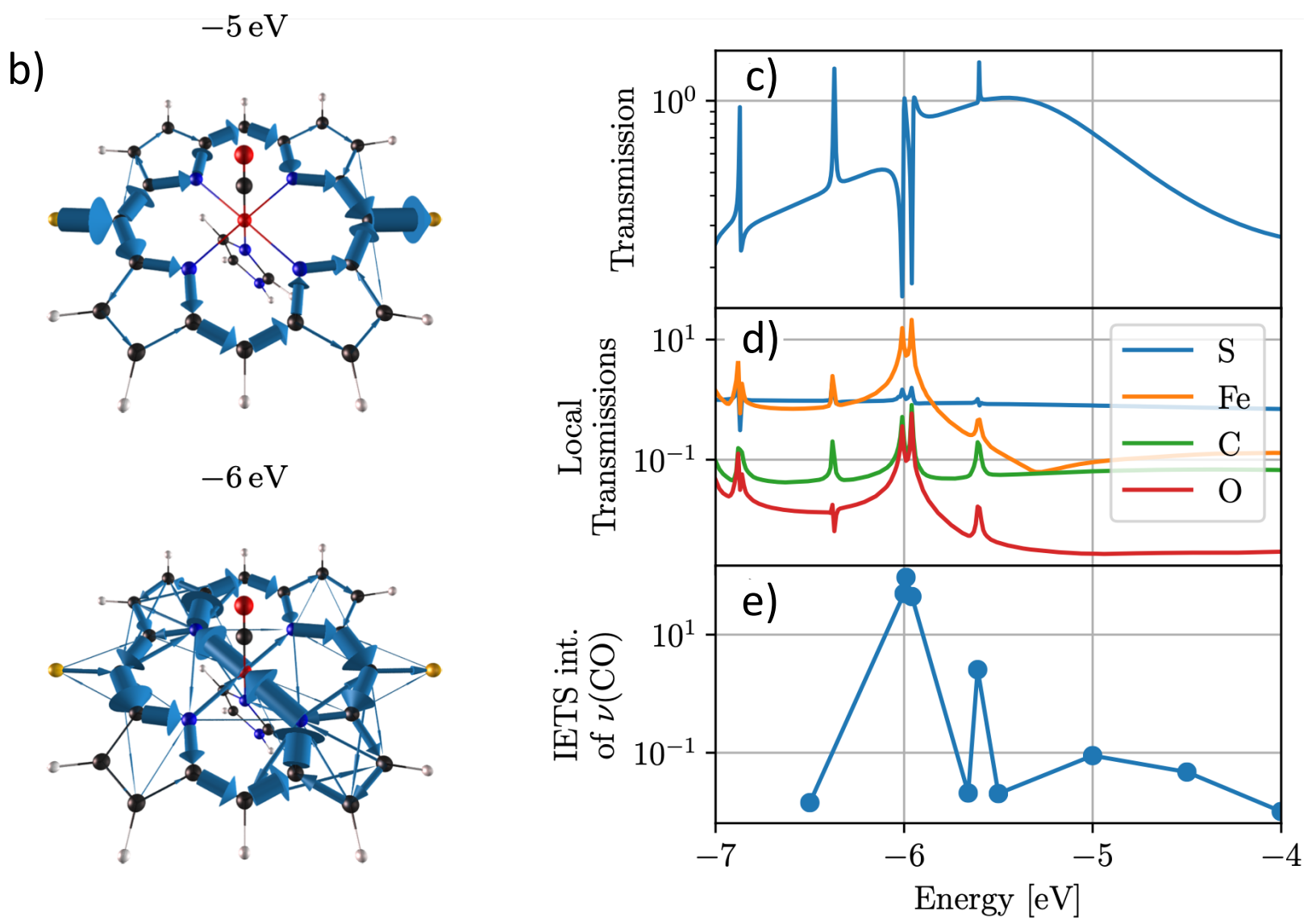

Figure 2: a) Lewis structure for the iron-porphyrin wire. b) Local transmissions at -5.0 and $-6.0 \mathrm{eV}$. c) Total transmission through the molecule. d) Local transmissions for an $\mathrm{S}$ atom from the anchoring group, the iron center and the adsorbed $\mathrm{CO}$ as obtained using equation (5). e) IETS intensity for the $\nu(\mathrm{CO})$ mode at different energies. The intensity of the $\mathrm{CO}$ mode correlates with the local transmissions through the $\mathrm{Fe} / \mathrm{CO}$ center.

If as here only certain (e.g., localized) vibrations are of interest, first-principles calculations of vibrational modes in the harmonic approximation can be greatly accelerated by selective techniques, which only evaluate the relevant modes (without loosing accuracy com- 
pared with a full calculation). This is particularly important for molecular junctions, where a molecular bridge is attached to two fragments representing electrodes, resulting in a fairly large system. This gain in efficiency is achieved by the mode-tracking protocol. $\underline{89}$. 91 We have therefore programmed an interface which allows for combining both full vibrational analyses and selective mode-tracking calculations with the evaluation of IETS intensities (see Supporting Information) and have applied it here to effectively evaluate the CO stretch vibration only, which takes 4 single point calculations to converge - in contrast to 324 for a full evaluation of the Hessian matrix. Since IETS calculations (and also experiments) can be quite sensitive to structural (and for the calculations, computational) parameters, we apply mode-tracking also for efficient validation of our methodology, which is also discussed in the Supporting Information.

Next, we study a nitrile-substituted biphenyl junction which was already suggested in Ref. ${ }^{59}$ as an example where IETS shows low intensities for the vibrations located on the CN substituents due to the current bypassing them. Our local transmission analysis confirms that for most energies (Figure 3), except for those associated with sharp destructive interference features, current indeed barely passes through the substituents: The summed-up local transmission through the carbon atoms between the benzene units is more than one order of magnitude higher than through the atoms of the nitrile groups (Figure $3 \mathrm{~b}$ ). This is in line with the IETS intensities for the two $\mathrm{C} \equiv \mathrm{N}$ stretching modes at $2241 \mathrm{~cm}^{-1}$ (asymmetric) and $2242 \mathrm{~cm}^{-1}$ (symmetric), which are much smaller than for the central $\mathrm{C} \equiv \mathrm{C}$ mode stretching mode at $\left(2209 \mathrm{~cm}^{-1}\right)$. Approaching energies where the interference features appear, the local transmissions resulting from ring currents through the nitrile groups become comparable to (or higher than) the transmission through the carbon atoms of the central linking unit, which is accompanied by a change in the ratio of IETS intensities. 
a)

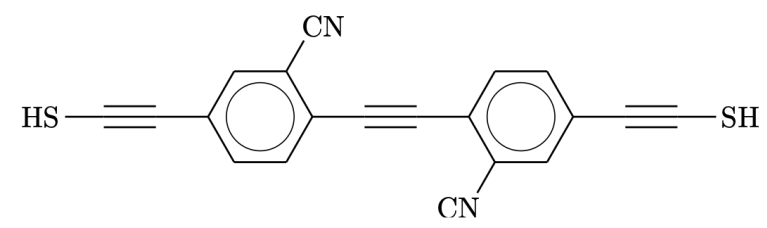

b)

c)
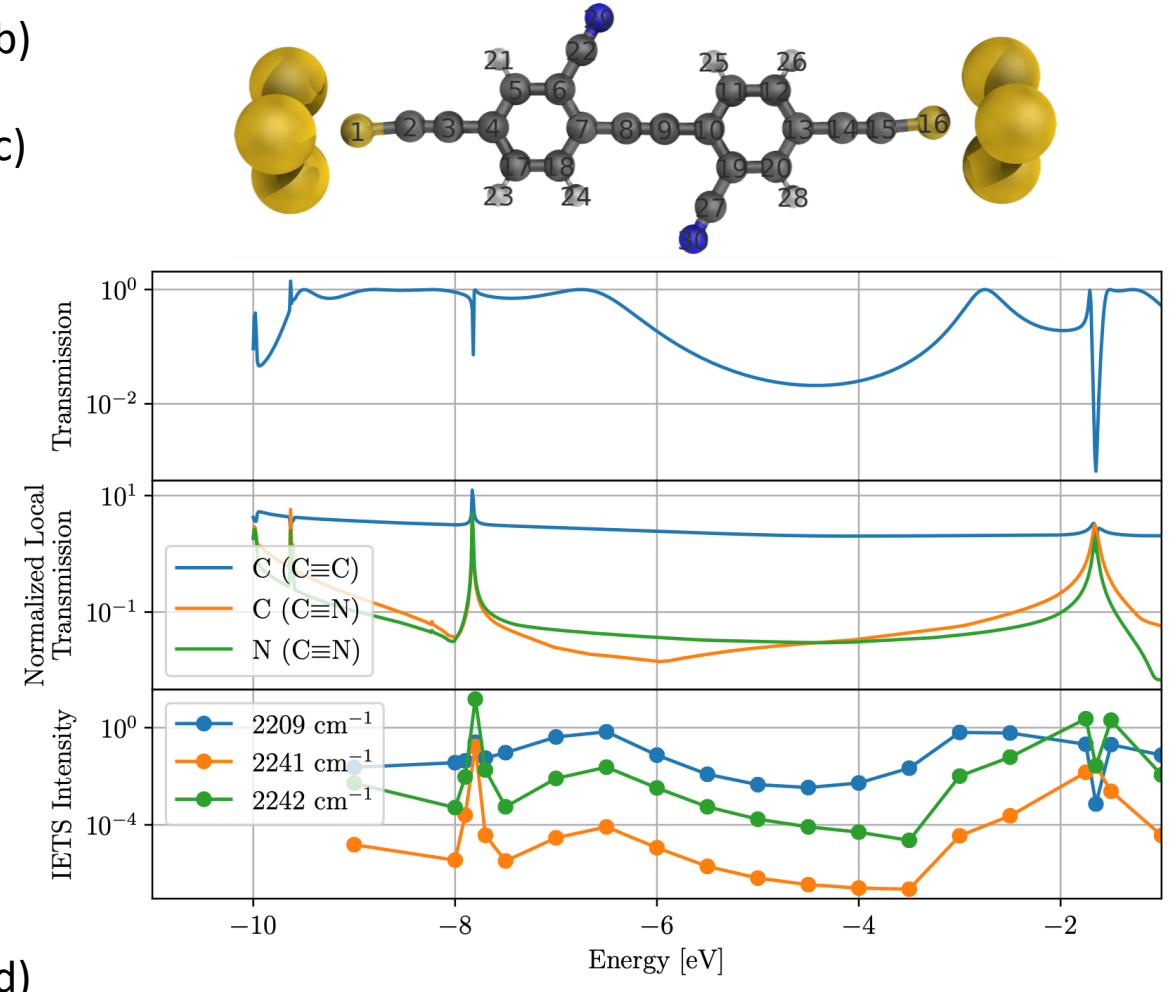

d)

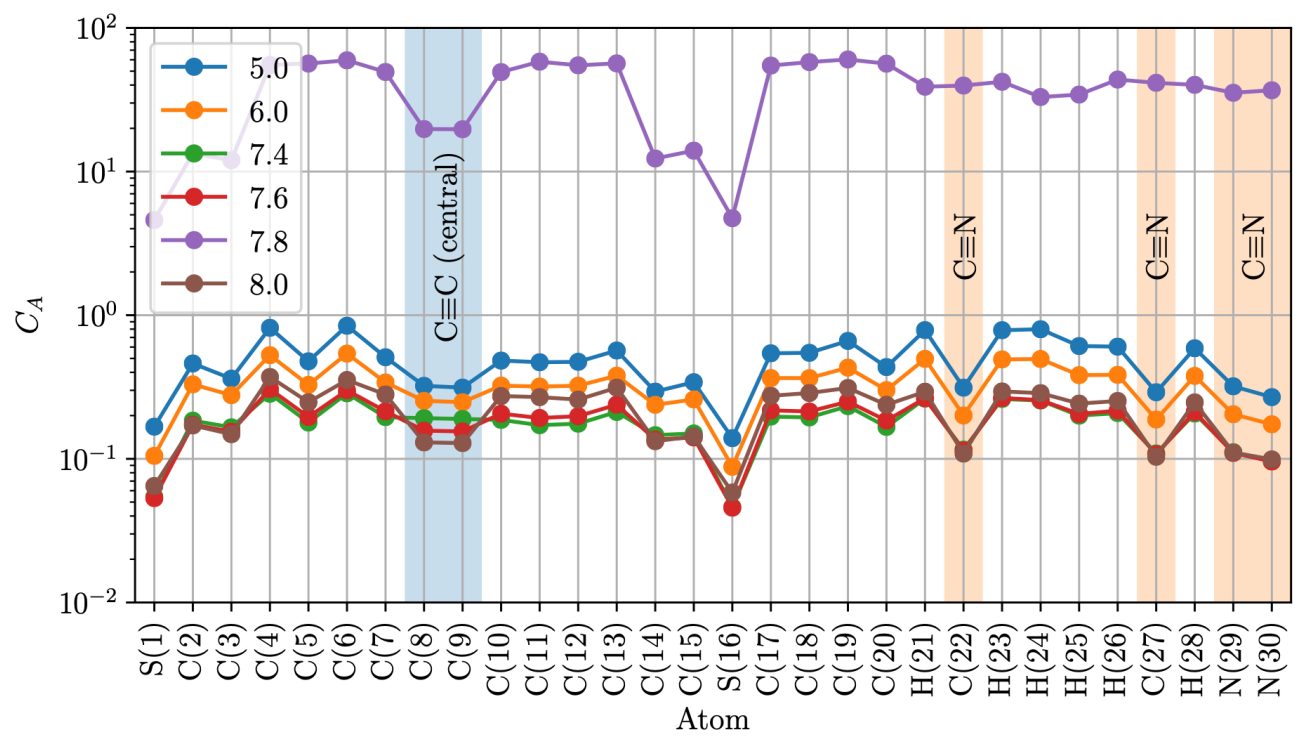

Figure 3: a) Lewis structure for the nitrile-substituted biphenyl molecule. b) Ball-andstick representation of the molecule attached to $\mathrm{Au}_{3}$ clusters. c) Transmission, normalized summed-up local transmissions (Equation (5) and IETS intensity of he stretching mode of the central $\mathrm{C} \equiv \mathrm{C}$ bond $\left(2209 \mathrm{~cm}^{-1}\right)$ and the antisymmetric and symmetric $\mathrm{C} \equiv \mathrm{N}$ stretching vibrations. d) Statistical analysis of the contribution of the individual atoms to the IETS (Equation (6); see b) for numbering scheme). Only modes with wavenumbers above $500 \mathrm{~cm}^{-1}$ were included. 
a)

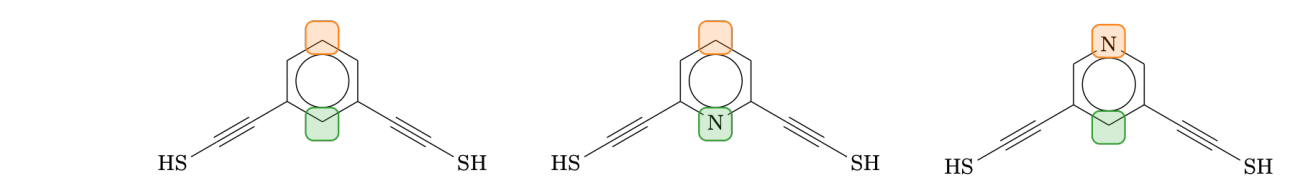

b)

c)
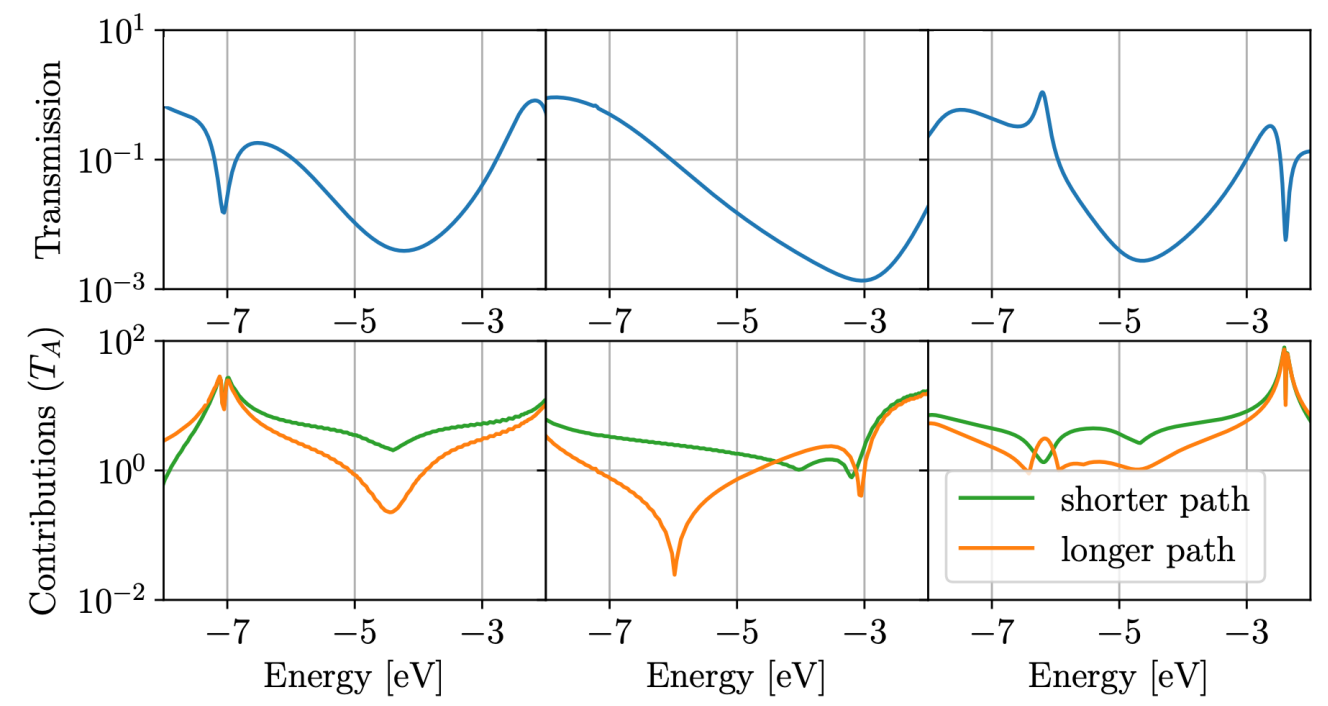

d)
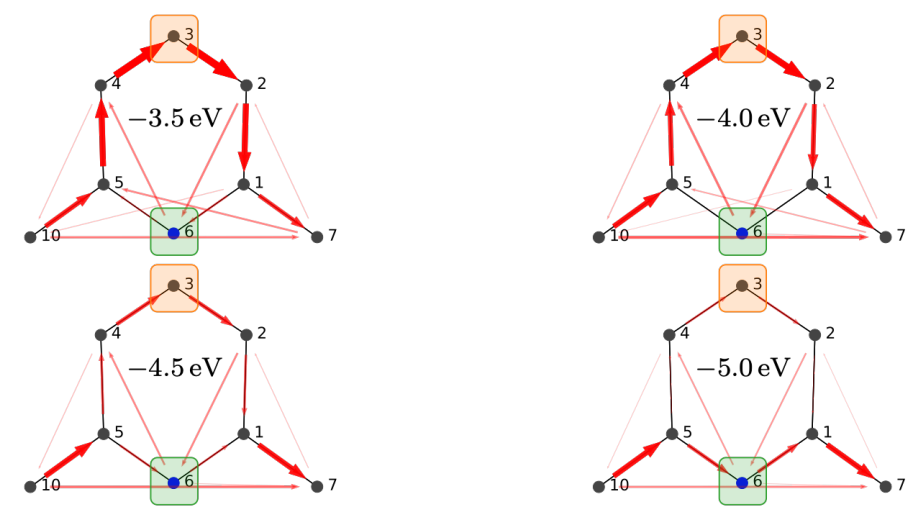

e)

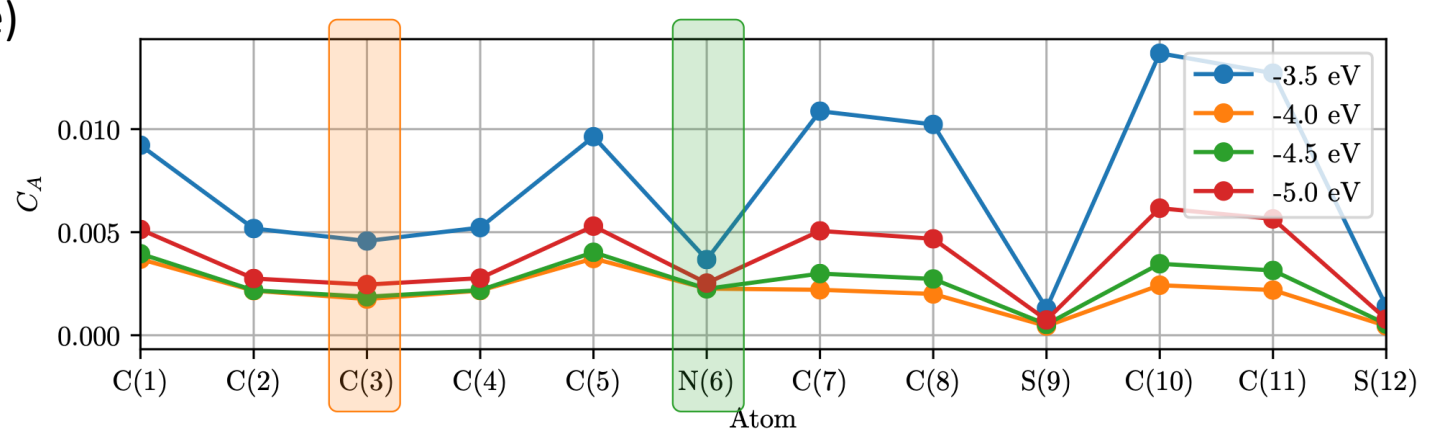

Figure 4: a) Lewis structures of meta-connected wires. b) Transmissions functions. c) Local transmissions through the upper/lower path, obtained by summing over all local transmissions $T_{A}$ from/to the marked atoms according to Equation (5). A consistency check for the middle molecule with the other atoms of the ring can be found in the Supporting Information. d) Local transmissions through the central ring (hydrogen atoms not shown) and e) summed contribution to IETS intensities (Equation (6) for the middle molecule (a)) at four different energies. The favored tunneling path changes from the upper to the lower part of the central ring. Atoms 8 and 9 (11 and 12) are not shown in d); they are the $\mathrm{C}$ and $\mathrm{S}$ atoms attached to 7 (10). 
In the structure just discussed, the $\mathrm{C} \equiv \mathrm{N}$ vibrational modes are still fairly localized, but less so than in the previous example involving the CO ligand (see Supporting Information). Still, our analysis suggests that the correlation between IETS intensities and pathways still holds. As a final example, we will study three structures based on a meta-connected benzene wire (Figure 4 left), and two pyridine analogs differing in the location of the nitrogen atom in the ring (middle and right). Conductance in such structures is reduced by destructive quantum interferences, and inelastic contributions are larger than for molecules without interference. ${ }^{6019293}$ The presence or absence of the nitrogen atom in the two different pathways around the ring should (and does) lead to differences in the preference of the tunneling electron for which pathway it takes.

Off resonance, for the benzene junction the short path is preferred over to longer one, as can be seen in the left panel of Figure $4 \mathrm{c}$ ), since the ratio of local contributions through the lower (green) vs the upper (orange) carbon atom is always larger than one. Inserting a nitrogen atom into the long path does not change the situation substantially (except for energies of around $-6 \mathrm{eV}$, right panel). By insertion of the nitrogen in the shorter path (middle panel), a clear crossover at $-4.78 \mathrm{eV}$ can be observed. At higher energies (up to about $-3 \mathrm{eV})$, the longer path is preferred, while at lower energies the electron takes the shorter path. Thus, when varying the Fermi level, we would hope to see a similar change of ratio of the IETS intensities.

Due to their delocalized nature (see Supporting Information), the vibrational modes of the molecule can not be easily assigned to fragments of the molecule corresponding to tunneling pathways, so we evaluate a weighted sum of the IETS peaks for each atom (inspired by Ref. $\left.{ }^{[52}\right)$,

$$
C_{A}=\sum_{\alpha}^{N_{Q}}\left|d_{A, Q_{\alpha}}\right| W_{\alpha}
$$

where $N_{Q}$ is the number of vibrational modes, and $\left|d_{A, Q_{\alpha}}\right|$ is the length of the displacement 
vector for the $x, y, z$ degrees of freedom of atom $A$ in mode $Q_{\alpha}$. Thus, if an atom is participating in a vibrational mode which has a high IETS intensity, this would significantly add to $C_{A}$. The full IET spectra can be found in the SI.

Figure (4) shows the calculated atomic IETS contributions close to the crossing point discussed above. We would expect the contribution of the nitrogen atom (marked green in the Figures) to decrease with energy in comparison to the contribution through the topmost carbon atom (marked orange). This trend cannot be seen in the in the plot. The IETS intensities in general drop from $E_{\mathrm{F}}=-5$ to $-4.0 \mathrm{eV}$, and rise when going to $-3.5 \mathrm{eV}$. In all cases, the ratio between the contribution of the nitrogen atom and the top carbon atom stays constant, only at $-3.5 \mathrm{eV}$ a slight relative drop of the contributions of the nitrogen atom can be observed. This would be in line with the analysis of the local transmissions, but is to weak to draw conclusions.

The low contributions of the sulfur atoms might be puzzling at first, since more or less all of the current has to enter/leave the system through these atoms. They contribute mostly to modes with low wavenumbers $\left(<500 \mathrm{~cm}^{-1}\right)$. These wavenumbers were left out of the analysis since the sulfur-gold interface, not being our focus, is not described very accurately by the attachment to gold clusters without further structural relaxations, and since lowwavenumber vibrations are not described well by the harmonic approximation. Also, most molecular vibrations are at higher wavenumbers (even though exceptions may exist, see e.g. Ref. ${ }^{(94)}$.

As a consistency check, we also perform the statistical analysis for the CN-substituted biphenyl (Figure $3 \mathrm{~d}$ ), note that due to the larger variations in $C_{A}$ for this molecule, a logarithmic scale was chosen). Regardless of the choice of Fermi energy, the summed-up IETS contributions $C_{A}$ for the $\mathrm{CN}$ atoms are consistently lower than for the carbon atoms making up the biphenyl backbone (as above, the sulfur atoms have low contributions because the lower-frequency vibrations were not taken into account in the summation in Eq. (5)). The only exception is in at the sharp antiresonance feature $(-7.8 \mathrm{eV})$, which involves local 
transmissions through the CN groups, and accordingly has larger $T_{A}$ on those atoms. This statistical analysis is less clear-cut than the considerations based on selected vibrational modes discussed above, but importantly, it supports the same conclusion on the ability of IETS to extract information on electron tunneling pathways in this system.

Overall, our results suggest that in systems where the vibrational degrees of freedom are strongly delocalized, it is difficult to deduce tunneling pathways using IETS. This conclusion could also be a due to approximations inherent in the theoretical methods employed here, for example there are cases in which currents do not follow bonds ${ }^{51}$ (as they are forced to do here), which would require more flexible theoretical approaches as described in, e.g., Refs. $\frac{4549}{51}$ Yet, these cases so far have been limited to aliphatic compounds, $\frac{51}{5}$ and the past success of atom-to-atom pathway analysis for describing and elucidating IET spectra and single-molecule conductance properties of conjugated systems as studied here suggests that our conclusion is reasonable, and that if a correlation between electron transport pathways and IET spectra for molecules with delocalized vibrational modes can be made, it is at least not straightforward.

\section{Methods}

Molecules were structurally optimized as dithiols using TURBOMOLE $6.6,{ }^{95}$ Ahlrichs' splitvalence triple- $\zeta$ basis set with polarization functions on all atoms (def2-TZVP 99100 ) and the BP86 exchange-correlation functional. ${ }^{101102}$ We used the resolution-of-identity (RI) approximation as implemented in TuRBOmole 6.6.103.105 The convergence criterion of the self-consistent field (SCF) algorithm was a change of energy below $10^{-8}$ Hartree. The convergence criterion for the structure optimizations was a gradient below $10^{-4}$ atomic units. To mimic the electrodes, after the optimization of the molecules, $\mathrm{Au}_{3}$ clusters were attached to the terminal sulfur atoms after stripping off the terminal hydrogen atoms, mimicking hollow-site adsorption on $\mathrm{Au}(111)$ surfaces. The sulfur-gold distance was $2.85 \AA$. Vibra- 
tional analyses were performed in the harmonic approximation using MoVIPAC, $\underline{91106}$ employing BP86/def2-TZVP on the cluster-molecule-cluster structures. This is reasonable even though the molecule-metal interfaces were not structurally optimized (to prevent unphysical distortions resulting from the small cluster size), as it was shown for thiolate molecules on silver clusters. $\stackrel{107}{ }$ For both full vibrational analyses and selective calculations via modetracking, second derivatives of the total energy w.r.t. structural displacements were evaluated in a seminumerical fashion (such that each second derivative needs two single-point calculations $\left.{ }^{89108}\right)$. If the mode-tracking algorithm ${ }^{8990}$ was applied to selectively calculate a specific vibrational normal mode, the convergence criterion was set to 0.0005 for the maximum component of the residuum vector, for root homing the overlap with the last selected eigenvector was used as a selection criterion, and for preconditioning the backtransformation of the Davidson matrix was employed. Transport calculations were performed by carrying out single-point DFT calculations (B3LYP109/111/LanL2DZ) with GAUSSIAN 09 112 on the cluster-molecule-cluster structures, followed by postprocessing via ARTAIOs. $\frac{172113}{\text { This }}$ smaller basis set was employed to prevent ghost transmission. ${ }^{72}$ The wide-band limit was used to obtain the Green's function matrices of the isolated electrodes, with the constant LDOS set to $0.036 \mathrm{eV}^{-1}$ as calculated by DFT for the $6 s$ band of gold. $\stackrel{114}{ }$ For IETS calculations, the same electronic structure settings were used as for the transport calculations. The details of the implementation using Artaios and MoViPAC can be found in the Supporting Information.

\section{Acknowledgments}

This work was supported by the Cluster of Excellence "Advanced Imaging of Matter" of the Deutsche Forschungsgemeinschaft (DFG) - EXC 2056 — project ID 390715994. The authors thank Martin Sebastian Zöllner for helpful discussions. 


\section{References}

(1) Cuevas, J. C.; Scheer, E. In Molecular Electronics: An Introduction to Theory and Experiment, 2nd ed.; Reed, M., Reed, M., Eds.; World Scientific Series in Nanotechnology and Nanoscience; World Scientific: Singapore, 2017; Vol. 1.

(2) Xiang, D.; Wang, X.; Jia, C.; Lee, T.; Guo, X. Molecular-Scale Electronics: From Concept to Function. Chem. Rev. 2016, 116, 4318-4440.

(3) Metzger, R. M. Unimolecular Electronics. Chem. Rev. 2015, 115, 5056-5115.

(4) Su, T. A.; Neupane, M.; Steigerwald, M. L.; Venkataraman, L.; Nuckolls, C. Chemical principles of single-molecule electronics. Nature Rev. Mater. 2016, 1, 16002.

(5) Ratner, M. A. A brief history of molecular electronics. Nature Nanotech. 2013, 8, $378-381$.

(6) Nitzan, A. Electron Transmission through Molecules and Molecular Interfaces. Annu. Rev. Phys. Chem. 2001, 52, 681-750.

(7) Nichols, R. J.; Higgins, S. J. Single-Molecule Electronics: Chemical and Analytical Perspective. Annu. Rev. Anal. Chem. 2015, 8, 389-417.

(8) Lörtscher, E. Wiring molecules into circuits. Nature Nanotechnol. 2013, 8, 381-384.

(9) Solomon, G. C.; Herrmann, C.; Ratner, M. A. Molecular Electronic Junction Transport: Some Pathways and Some Idea. Top. Curr. Chem. 2012, 313, 1-38.

(10) Komoto, Y.; Fujii, S.; Iwane, M.; Kiguchi, M. Single-molecule junctions for molecular electronics. J. Mater. Chem. C 2016, 4, 8842-8858.

(11) Jeong, H.; Kim, D.; Xiang, D.; Lee, T. High-Yield Functional Molecular Electronic Devices. ACS Nano 2017, 11, 6511-6548. 
(12) Sun, L.; Diaz-Fernandez, Y. A.; Gschneidtner, T. A.; Westerlund, F.; Lara-Avila, S.; Moth-Poulsen, K. Single-molecule electronics: from chemical design to functional devices. Chem. Soc. Rev. 2014, 43, 7378-7411.

(13) Balabin, I. A.; Onuchic, J. N. Dynamically Controlled Protein Tunneling Paths in Photosynthetic Reaction Centers. Science 2000, 290, 114-117.

(14) Beratan, D. N.; Balabin, I. A. Heme-copper oxidases use tunneling pathways. Proc. Nat. Acad. Sci. 2008, 105, 403-404.

(15) Balabin, I. A.; Hu, X.; Beratan, D. N. Exploring Biological Electron Transfer Pathway Dynamics with the Pathways Plugin for VMD. J. Comput. Chem. 2012, 33, 906-910.

(16) Jasaitis, A.; Johansson, M. P.; Wikstrom, M.; Vos, M. H.; Verkhovsky, M. I. Nanosecond electron tunneling between the hemes in cytochrome bo3. Proc. Nat. Acad. Sci. 2007, 104, 20811-20814.

(17) Ullmann, G. M.; Kostic, N. M. Electron-Tunneling Paths in Various Electrostatic Complexes between Cytochrome c and Plastocyanin. Anisotropy of the Copper-Ligand Interactions and Dependence of the Iron-Copper Electronic Coupling on the Metalloprotein Orientation. J. Am. Chem. Soc. 1995, 117, 4766-4774.

(18) Delor, M.; Scattergood, P. A.; Sazanovich, I. V.; Parker, A. W.; Greetham, G. M.; Meijer, A. J. H. M.; Towrie, M.; Weinstein, J. A. Toward control of electron transfer in donor-acceptor molecules by bond-specific infrared excitation. Science 2014, 346, 1492-1495.

(19) Tsuji, Y.; Hoffmann, R. Helical Oligoenes: Conformations, Bond Alternation, and Competing Through-Bond and Through-Space Transmission. Chem. Eur. J. 2016, 22, 4878-4888. 
(20) Garner, M. H.; Jensen, A.; Hyllested, L. O. H.; Solomon, G. C. Helical orbitals and circular currents in linear carbon wires. Chem. Sci. 2019, 10, 4598-4608.

(21) Naaman, R.; Paltiel, Y.; Waldeck, D. H. Chiral molecules and the electron spin. Nature Reviews 2019, 3, 250-260.

(22) Abendroth, J. M.; Cheung, K. M.; Stemer, D. M.; Hadri, M. S. E.; Zhao, C.; Fullerton, E. E.; Weiss, P. S. Spin-Dependent Ionization of Chiral Molecular Films. J. Am. Chem. Soc. 2019, 141, 3863-3874.

(23) Xie, Z.; Markus, T. Z.; Cohen, S. R.; Vager, Z.; Gutierrez, R.; Naaman, R. Spin Specific Electron Conduction through DNA Oligomers. Nano Lett. 2011, 11, 4652-4655.

(24) Zwang, T. J.; Hürlimann, S.; Hill, M. G.; Barton, J. K. Helix-Dependent Spin Filtering through the DNA Duplex. J. Am. Chem. Soc. 2016, 138, 15551-15554.

(25) Göhler, B.; Hamelbeck, V.; Markus, T. Z.; Kettner, M.; Hanne, G. F.; Vager, Z.; Naaman, R.; Zacharias, H. Spin selectivity in electron transmission through self-assembled monolayers of double-stranded DNA. Science 2011, 331, 894-897.

(26) Mishra, D.; Markus, T. Z.; Naaman, R.; Kettner, M.; Göhler, B.; Zacharias, H.; Friedman, N.; Sheves, M.; Fontanesi, C. Spin-dependent electron transmission through bacteriorhodopsin embedded in purple membrane. Proc. Natl. Acad. Sci. 2013, 110, $14872-14876$.

(27) Aragones, A. C. et al. Measuring the Spin-Polarization Power of a Single Chiral Molecule. small 2017, 13, 1602519.

(28) Kettner, M. et al. Spin Filtering in Electron Transport Through Chiral Oligopeptides. J. Phys. Chem. C 2015, 119, 14542-14547.

(29) Kettner, M.; Maslyuk, V. V.; Nürenberg, D.; Seibel, J.; Gutierrez, R.; Cuniberti, G.; 
Ernst, K.-H.; Zacharias, H. Chirality-Dependent Electron Spin Filtering by Molecular Monolayers of Helicenes. J. Phys. Chem. Lett. 2018, 9, 2025-2030.

(30) Kiran, V.; Mathew, S. P.; Cohen, S. R.; Delgado, I. H.; Lacour, J.; Naaman, R. Helicenes - A New Class of Organic Spin Filter. Adv. Mater. 2016, 28, 1957-1962.

(31) Varela, S.; Mujica, V.; Medina, E. Effective spin-orbit couplings in an analytical tightbinding model of DNA: spin filtering and chiral spin transport. Phys. Rev. B 2016, 93, 155436.

(32) Varela, S.; Mujica, V.; Medina, E. Spin-orbit Coupling Modulation in DNA by Mechanical Deformations. Chimia 2018, 72, 411-417.

(33) Maslyuk, V. V.; Gutierrez, R.; Dianat, A.; Mujica, V.; Cuniberti, G. Enhanced Magnetoresistance in Chiral Molecular Junctions. J. Phys. Chem. Lett. 2018, 9, 5453-5459.

(34) Guo, A.-M.; feng Sun, Q. Spin-Selective Transport of Electrons in DNA Double Helix. Phys Rev. Lett. 2012, 108, 218102.

(35) Gutierrez, R.; Diaz, E.; Naaman, R.; Cuniberti, G. Spin-selective transport through helical molecular systems. Phys. Rev. B 2012, 85, 081404.

(36) Gutierrez, R.; Diaz, E.; Gaul, C.; Brumme, T.; Dominguez-Adame, F.; Cuniberti, G. Modeling Spin Transport in Helical Fields: Derivation of an Effective Low-Dimensional Hamiltonian. J. Phys. Chem. C 2013, 117, 22276-22284.

(37) Zöllner, M. S.; Varela, S.; Medina, E.; Mujica, V.; Herrmann, C. Chiral-Induced Spin Selectivity: A Symmetry Analysis of Electronic Transmission. 2019; ChemRxiv, DOI: 10.26434/chemrxiv.8325248.v2.

(38) Guo, A.-M.; Sun, Q.-F. Spin-dependent electron transport in protein-like single-helical molecules. Proc. Natl. Acad. Sci. 2014, 111, 11658-11662. 
(39) Solomon, G. C.; Herrmann, C.; Hansen, T.; Mujica, V.; Ratner, M. A. Exploring local currents in molecular junctions. Nature Chemistry 2010, 2, 223-228.

(40) Dhakal, U.; Rai, D. Circular current and induced force in a molecular ring junction. J. Phys.: Condens. Matter 2019, 31, 125302.

(41) Rai, D.; Hod, O.; Nitzan, A. Circular Currents in Molecular Wires. The Journal of Physical Chemistry C 2010, 114, 20583-20594.

(42) Gryn'ova, G.; Corminboeuf, C. Topology-Driven Single-Molecule Conductance of Carbon Nanothreads. J. Phys. Chem. Lett. 2019, 10, 825-830.

(43) Herrmann, C.; Solomon, G. C.; Ratner, M. A. Local Pathways in Coherent Electron Transport through Iron Porphyrin Complexes: A Challenge for First-Principles Transport Calculations. The Journal of Physical Chemistry C 2010, 114, 20813-20820.

(44) Hansen, T.; Solomon, G. C.; Hansen, T. Interatomic inelastic current. J. Chem. Phys. 2017, 146, 092322 .

(45) Sai, N.; Bushong, N.; Hatcher, R.; di Ventra, M. Microscopic current dynamics in nanoscale junctions. Phys. Rev. B 2007, 75, 15410-115418.

(46) Ernzerhof, M.; Bahmann, H.; Goyer, F.; Zhuang, M.; Rocheleau, P. Electron Transmission through Aromatic Molecules. J. Chem. Theory Comput. 2006, 2, 1291-1297.

(47) Todorov, T. N. Tight-binding simulation of current-carrying nanostructures. J. Phys.: Condens. Matter 2002, 14, 3049-3084.

(48) Pecchia, A.; Carlo, A. D. Atomistic theory of transport in organic and inorganic nanostructures. Rep. Prog. Phys. 2004, 67, 1497-1561.

(49) Pohl, V.; Steinkasserer, L. E. M.; Tremblay, J. C. Imaging Time-Dependent Electronic Currents through a Graphene-Based Nanojunction. The Journal of Physical Chemistry Letters 2019, 10, 5387-5394. 
(50) Pohl, V.; Steinkasserer, L. E. M.; Tremblay, J. C. Imaging Time-Dependent Electronic Currents through a Graphene-Based Nanojunction. 2019.

(51) Jensen, A.; Garner, M. H.; Solomon, G. C. When Current Does Not Follow Bonds: Current Density in Saturated Molecules. J. Phys. Chem. C 2019, 123, 12042-12051.

(52) Troisi, A.; Beebe, J. M.; Picraux, L. B.; van Zee, R. D.; Stewart, D. R.; Ratner, M. A.; Kushmerick, J. G. Tracing electronic pathways in molecules by using inelastic tunneling spectroscopy. Proceedings of the National Academy of Sciences 2007, 104, 1425514259 .

(53) Troisi, A.; Ratner, M. A. Inelastic insights for molecular tunneling pathways: Bypassing the terminal groups. Physical Chemistry Chemical Physics 2007, 9, 2421.

(54) Galperin, M.; Ratner, M. A.; Nitzan, A. Molecular transport junctions: vibrational effects. Journal of Physics: Condensed Matter 2007, 19, 103201.

(55) Reed, M. A. Inelastic electron tunneling spectroscopy. Materials Today 2008, 11, $46-50$.

(56) Jaklevic, R. C.; Lambe, J. Molecular Vibration Spectra by Electron Tunneling. Physical Review Letters 1966, 17, 1139-1140.

(57) Paulsson, M.; Frederiksen, T.; Ueba, H.; Lorente, N.; Brandbyge, M. Unified Description of Inelastic Propensity Rules for Electron Transport through Nanoscale Junctions. Physical Review Letters 2008, 100.

(58) Gagliardi, A.; Solomon, G. C.; Pecchia, A.; Frauenheim, T.; Carlo, A. D.; Hush, N. S.; Reimers, J. R. A priorimethod for propensity rules for inelastic electron tunneling spectroscopy of single-molecule conduction. Physical Review B 2007, 75.

(59) Troisi, A.; Ratner, M. A. Molecular Transport Junctions: Propensity Rules for Inelastic Electron Tunneling Spectra. Nano Letters 2006, 6, 1784-1788. 
(60) Lykkebo, J.; Gagliardi, A.; Pecchia, A.; Solomon, G. C. IETS and quantum interference: Propensity rules in the presence of an interference feature. The Journal of Chemical Physics 2014, 141, 124119.

(61) Fung, E.-D.; Gelbwaser, D.; Taylor, J.; Low, J.; Xia, J.; Davydenko, I.; Campos, L. M.; Marder, S.; Peskin, U.; Venkataraman, L. Breaking Down Resonance: Nonlinear Transport and the Breakdown of Coherent Tunneling Models in Single Molecule Junctions. Nano Lett. 2019, 19, 2555-2561.

(62) Chalmers, J.; Griffiths, P. Handbook of vibrational spectroscopy; Handbook of Vibrational Spectroscopy Bd. 1; J. Wiley, 2002; Vol. 1: Theory and Instrumentation.

(63) Zandvliet, H. J.; van Houselt, A. Scanning Tunneling Spectroscopy. Annual Review of Analytical Chemistry 2009, 2, 37-55.

(64) Paulsson, M.; Frederiksen, T.; Brandbyge, M. Inelastic Transport through Molecules: Comparing First-Principles Calculations to Experiments. Nano Lett. 2006, 6, 258262.

(65) Lü, J.-T.; Christensen, R. B.; Foti, G.; Frederiksen, T.; Gunst, T.; Brandbyge, M. Efficient calculation of inelastic vibration signals in electron transport: Beyond the wide-band approximation. Physical Review B 2014, 89.

(66) Chen, Y.-C.; Zwolak, M.; Di Ventra, M. Inelastic Current-Voltage Characteristics of Atomic and Molecular Junctions. Nano Letters 2004, 4, 1709-1712.

(67) Solomon, G. C.; Gagliardi, A.; Pecchia, A.; Frauenheim, T.; Carlo, A. D.; Reimers, J. R.; Hush, N. S. Understanding the inelastic electron-tunneling spectra of alkanedithiols on gold. The Journal of Chemical Physics 2006, 124, 094704.

(68) Jiang, J.; Kula, M.; Lu, W.; Luo, Y. First-Principles Simulations of Inelastic Elec- 
tron Tunneling Spectroscopy of Molecular Electronic Devices. Nano Letters 2005, 5, $1551-1555$.

(69) Troisi, A.; Ratner, M. A.; Nitzan, A. Vibronic effects in off-resonant molecular wire conduction. The Journal of Chemical Physics 2003, 118, 6072-6082.

(70) Caroli, C.; Combescot, R.; Nozieres, P.; Saint-James, D. Direct calculation of the tunneling current. J. Phys. C: Solid State Phys. 1971, 4, 916-929.

(71) Mujica, V.; Kemp, M.; Ratner, M. A. Electron conduction in molecular wires. I. A scattering formalism. J. Chem. Phys. 1994, 101, 6849.

(72) Herrmann, C.; Solomon, G. C.; Subotnik, J. E.; Mujica, V.; Ratner, M. A. Ghost transmission: How large basis sets can make electron transport calculations worse. The Journal of Chemical Physics 2010, 132, 024103.

(73) Xue, Y.; Datta, S.; Ratner, M. A. First-principles based matrix Green's function approach to molecular electronic devices: general formalism. Chemical Physics 2002, 281, 151-170.

(74) Troisi, A. Inelastic electron tunnelling in saturated molecules with different functional groups: correlations and symmetry considerations from a computational study. $J$. Phys.: Condens. Matter 2008, 20, 374111.

(75) Troisi, A.; Ratner, M. A. Modeling the inelastic electron tunneling spectra of molecular wire junctions. Physical Review B 2005, 72.

(76) Arroyo, C. R.; Tarkuc, S.; Frisenda, R.; Seldenthuis, J. S.; Woerde, C. H. M.; Eelkema, R.; Grozema, F. C.; van der Zant, H. S. J. Signatures of Quantum Interference Effects on Charge Transport Through a Single Benzene Ring. Angewandte Chemie International Edition 2013, 52, 3152-3155. 
(77) Su, T. A.; Neupane, M.; Steigerwald, M. L.; Venkataraman, L.; Nuckolls, C. Chemical principles of single-molecule electronics. Nature Reviews Materials 2016, 1.

(78) Stuyver, T.; Blotwijk, N.; Fias, S.; Geerlings, P.; De Proft, F. Exploring Electrical Currents through Nanographenes: Visualization and Tuning of the through-Bond Transmission Paths. ChemPhysChem 2017, 18, 3012-3022.

(79) Garner, M. H. et al. Comprehensive suppression of single-molecule conductance using destructive $\sigma$-interference. Nature 2018, 558, 415-419.

(80) Garner, M. H.; Koerstz, M.; Jensen, J. H.; Solomon, G. C. The Bicyclo [2.2. 2] octane Motif: A Class of Saturated Group 14 Quantum Interference Based Single-molecule Insulators. J. Phys. Chem. Lett. 2018, 9, 6941-6947.

(81) Schlicke, H.; Herrmann, C. Controlling molecular conductance: switching off $\pi$ sites through protonation. ChemPhysChem 2014, 15, 4011-4018.

(82) Hannewald, C. D. N. Organic/Inorganic Hybrid Materials: Challenges for ab Initio Methodology. Acc. Chem. Res. 2014, 47, 3225-3232.

(83) Egger, D. A.; Liu, Z.-F.; Neaton, J. B.; Kronik, L. Reliable Energy Level Alignment at Physisorbed Molecule-Metal Interfaces from Density Functional Theory. Nano Lett. 2015, 15, 2448-2455.

(84) Venkataraman, L.; Park, Y. S.; Whalley, A. C.; Nuckolls, C.; Hybertsen, M. S.; Steigerwald, M. L. Electronics and Chemistry: Varying Single-Molecule Junction Conductance Using Chemical Substituents. Nano Lett. 2007, 7, 502-506.

(85) Herrmann, C.; Solomon, G. C.; Ratner, M. A. Organic radicals as spin filters. J. Am. Chem. Soc. 2010, 132, 3682-3684.

(86) Saito, S.; Osuka, A. Expanded Porphyrins: Intriguing Structures, Electronic Properties, and Reactivities. Angewandte Chemie International Edition 2011, 50, 4342-4373. 
(87) Auwärter, W.; Seufert, K.; Bischoff, F.; Ecija, D.; Vijayaraghavan, S.; Joshi, S.; Klappenberger, F.; Samudrala, N.; Barth, J. V. A surface-anchored molecular four-level conductance switch based on single proton transfer. Nature Nanotechnology 2011, 7, $41-46$.

(88) Stuyver, T.; Perrin, M.; Geerlings, P.; Proft, F. D.; Alonso, M. Conductance Switching in Expanded Porphyrins through Aromaticity and Topology Changes. Journal of the American Chemical Society 2018, 140, 1313-1326.

(89) Reiher, M.; Neugebauer, J. A mode-selective quantum chemical method for tracking molecular vibrations applied to functionalized carbon nanotubes. The Journal of Chemical Physics 2003, 118, 1634-1641.

(90) Herrmann, C.; Neugebauer, J.; Reiher, M. Finding a needle in a haystack: direct determination of vibrational signatures in complex systems. New Journal of Chemistry 2007, 31, 818 .

(91) Weymuth, T.; Haag, M. P.; Kiewisch, K.; Luber, S.; Schenk, S.; Jacob, C. R.; Herrmann, C.; Neugebauer, J.; Reiher, M. MOVIPAC: Vibrational spectroscopy with a robust meta-program for massively parallel standard and inverse calculations. Journal of Computational Chemistry 2012, 33, 2186-2198.

(92) Lykkebo, J.; Gagliardi, A.; Pecchia, A.; Solomon, G. C. Strong Overtones Modes in Inelastic Electron Tunneling Spectroscopy with Cross-Conjugated Molecules: A Prediction from Theory. ACS Nano 2013, 7, 9183-9194.

(93) Salhani, C.; Rocca, M. L. D.; Bessis, C.; Bonnet, R.; Barraud, C.; Lafarge, P. Inelastic electron tunneling spectroscopy in molecular junctions showing quantum interference. Phys. Rev. B 2017, 95, 165431.

(94) Chen, H. et al. Evidence for Ultralow-Energy Vibrations in Large Organic Molecules. Nano Lett. 2017, 17, 4929-4933. 
(95) TURBOMOLE V7.1, a development of University of Karlsruhe and Forschungszentrum Karlsruhe GmbH, 1989-2007, TURBOMOLE GmbH, since 2007; available from http://www.turbomole.com. 2016.

(96) Ahlrichs, R.; Bär, M.; Häser, M.; Horn, H.; Kölmel, C. Electronic structure calculations on workstation computers: The program system turbomole. Chem. Phys. Lett. 1989, 162, $165-169$.

(97) Treutler, O.; Ahlrichs, R. Efficient molecular numerical integration schemes. J. Chem. Phys. 1995, 102, 346-354.

(98) Von Arnim, M.; Ahlrichs, R. Performance of parallel TURBOMOLE for density functional calculations. J. Comput. Chem. 1998, 19, 1746-1757.

(99) Schäfer, A.; Huber, C.; Ahlrichs, R. Fully optimized contracted Gaussian basis sets of triple zeta valence quality for atoms Li to Kr. J. Chem. Phys. 1994, 100, 5829-5835.

(100) Weigend, F.; Ahlrichs, R. Balanced basis sets of split valence, triple zeta valence and quadruple zeta valence quality for $\mathrm{H}$ to Rn: Design and assessment of accuracy. Phys. Chem. Chem. Phys. 2005, 7, 3297-3305.

(101) Becke, A. D. Density-functional exchange-energy approximation with correct asymptotic behavior. Phys. Rev. A 1988, 38, 3098-3100.

(102) Perdew, J. P. Density-functional approximation for the correlation energy of the inhomogeneous electron gas. Phys. Rev. B 1986, 33, 8822-8824.

(103) Eichkorn, K.; Treutler, O.; Öhm, H.; Häser, M.; Ahlrichs, R. Auxiliary basis sets to approximate Coulomb potentials. Chem. Phys. Lett. 1995, 240, $283-290$.

(104) Eichkorn, K.; Weigend, F.; Treutler, O.; Ahlrichs, R. Auxiliary basis sets for main row atoms and transition metals and their use to approximate Coulomb potentials. Theor. Chem. Acc. 1997, 97, 119-124. 
(105) Weigend, F. Accurate Coulomb-fitting basis sets for H to Rn. Phys. Chem. Chem. Phys. 2006, 8, 1057-1065.

(106) Weymuth, T.; Haag, M. P.; Kiewisch, K.; Luber, S.; Schenk, S.; Jacob, C. R.; Herrmann, C.; Neugebauer, J.; Reiher, M. MoVIPAC - Vibrational Spectroscopy with a Robust Meta-Program for Massively Parallel Standard and Inverse Calculations, available via https://reiher.ethz.ch/software/movipac.html. 2012-2020.

(107) Herrmann, C.; Reiher, M. Direct targeting of adsorbate vibrations with mode-tracking. Surf. Sci. 2006, 9, 1891-1900.

(108) Neugebauer, J.; Reiher, M.; Kind, C.; Hess, B. A. Quantum Chemical Calculation of Vibrational Spectra of Large Molecules - Raman and IR Spectra for Buckminsterfullerene. J. Comput. Chem. 2002, 23, 895-910.

(109) Becke, A. D. Density-functional exchange-energy approximation with correct asymptotic behavior. Phys. Rev. A 1988, 38, 3098-3100.

(110) Lee, C.; Yang, W.; Parr, R. G. Development of the Colle-Salvetti correlation-energy formula into a functional of the electron density. Phys. Rev. B 1988, 37, 785-789.

(111) Becke, A. D. Density-functional thermochemistry. III. The role of exact exchange. J. Chem. Phys. 1993, 98, 5648-5652.

(112) Frisch, M. J. et al. Gaussian 09 Revision A.1. Gaussian Inc. Wallingford CT 2009.

(113) Deffner, M.; Gross, L.; Steenbock, T.; Voigt, B. A.; Solomon, G. C.; Herrmann, C. ARTAIOS - a transport code for postprocessing quantum chemical electronic structure calculations, available via https://www.chemie.unihamburg.de/institute/ac/arbeitsgruppen/herrmann/software/artaios.html. 20092020. 
(114) Papaconstantopoulos, D. A. Handbook of the Band Structure of Elemental Solids; Plenum: New York, 1986. 